\title{
Mechanisms of Enhanced Osteoclastogenesis in Alkaptonuria
}

Giacomina Brunetti, * Albina Tummolo, ${ }^{\dagger}$ Gabriele D’Amato, ${ }^{\dagger}$ Alberto Gaeta, ${ }^{\S}$ Federica Ortolani, ${ }^{\dagger}$ Laura Piacente, ${ }^{\top}$ Paola Giordano, "Silvia Colucci, ${ }^{*}$ Maria Grano, ${ }^{\|}$Francesco Papadia, ${ }^{\dagger}$ and Maria F. Faienza

From the Department of Basic Medical Sciences, Neuroscience and Sense Organs, * Section of Human Anatomy and Histology, the Department of Biomedical Sciences and Human Oncology, "Pediatric Section, and the Department of Emergency and Organ Transplantation," Section of Human Anatomy and Histology, University "Aldo Moro" of Bari, Bari; the Department of Metabolic Diseases, Clinical Genetics and Diabetology ${ }^{\dagger}$ and the Radiology Unit, Giovanni XXIII Children’s Hospital, Bari; and the Neonatal Intensive Care Unit, ${ }^{\ddagger}$ Di Venere Hospital, Bari, Italy

Accepted for publication December 19, 2017.

Address correspondence to Giacomina Brunetti, Ph.D., Department of Basic and Medical Sciences, Neurosciences and Sense Organs, Section of Human Anatomy and Histology, University "Aldo Moro" of Bari, Piazza Giulio Cesare, 11, 70124 Bari, Italy; or Maria F. Faienza, M.D., Department of Biomedical Science and Human Oncology, Pediatric Section, University "Aldo Moro" of Bari, Piazza G. Cesare, 11, 70124, Bari, Italy. E-mail: giacomina.brunetti@uniba.itor mariafelicia.faienza@uniba.it.

\begin{abstract}
Alkaptonuria (AKU) is a rare disorder characterized by the deficiency of the enzyme homogentisate 1,2-dioxygenase and consequent homogentisate accumulation, which leads to progressive and severe osteoarthopathy starting from the second decade of life. Thus, in AKU patients, bone involvement represents an important clinical issue, which we investigated. Serum levels of receptor activator of NF- $\kappa B$ ligand (RANKL), osteoprotegerin, sclerostin, Dickkopf-1, and bone remodeling markers were measured in nine AKU patients (two children and seven adults) and 22 controls, together with lumbar spine bone mineral density (LS-BMD) and femoral-BMD. In the two AKU children, the average of LS-BMD and femoralBMD Z-scores were within the normal range, but reduced with respect to the controls. Otherwise, in the adult AKU patients, LS-BMD T-score was inside the normal range, but femoral-BMD T-score reached osteopenic levels. Consistently, in AKU adults, higher RANKL and C-terminal telopeptide of collagen type 1 and lower osteoprotegerin levels were observed than in controls. Otherwise, spontaneous osteoclastogenesis was already evident in peripheral blood mononuclear cell cultures from AKU children, together with a high percentage of circulating osteoclast precursors. Osteoclastogenesis was sustained by the high levels of tumor necrosis factor- $\alpha$, RANK, RANKL, and LIGHT. In conclusion, the altered osteoclastogenesis was observed already in AKU children, despite the absence of evident injury. Thus, a preventive approach in young patients, targeting osteoclast activity, may prevent the macroscopic bone disease that appears in adult AKU. (Am J Pathol 2018, 188: 1059-1068; https://doi.org/10.1016/j.ajpath.2017.12.008)
\end{abstract}

Alkaptonuria (AKU), Online Mendelian Inheritance in Man number 203500 , is a rare metabolic disease attributable to a deficient activity of the enzyme homogentisate 1,2-dioxygenase, involved in Phe and Tyr catabolism. ${ }^{1}$ AKU subjects undergo accumulation of the homogentisic acid (HGA), which is prone to reactions of oxidation/polymerization, causing the production of a melanin-like pigment.

This represents a particular type of melanin, an HGA-melanin, found only in AKU patients. ${ }^{3}$ Recent studies have demonstrated that AKU osteoarticular tissue contains amyloid deposits and that alkaptonuric amyloid colocalized with HGA-melanin ochronotic pigment, as HGA, might act as an amyloid aggregation enhancer, thus mediating the osteoarthropathy damage. ${ }^{4,5}$ The progression of AKU, according to the chronology of appearance, is characterized by dark urine at birth, then ochronosis clinically visible in the skin and sclera at approximately 30 years, and finally a severe ochronotic arthropathy at approximately 40 to 50 years. ${ }^{4}$ Cardiovascular and renal complications have been described in numerous case report studies. ${ }^{6}$ It has been demonstrated that the progression of the joint damage in AKU patients, in association with extensive hyaline cartilage pigmentation, involves a remodeling of the calcified cartilage and underlying bone, leading to a phenotype similar to bone marrow lesions. ${ }^{4}$ In addition, an

Supported by Italian Ministry of Education, University and Research Ex (M.G. and S.C)

G.B. and A.T. contributed equally to this work.

Disclosures: None declared. 
aggressive resorption attributable to enhanced osteoclastic activity leads to complete loss of the subchondral plate. The cause of this particular phenotype is unknown, but it has been hypothesized that it may be a consequence of stress shielding because of the altered mechanical properties of the overlying hyaline articular cartilage. In fact, ochronotic pigment deposition increases the stiffness of the hyaline articular cartilage matrix, resulting in abnormal distribution of load through the tissues. ${ }^{7}$ Consistently with a high osteoclastic activity, an increase of bone resorption markers has been observed in AKU subjects. ${ }^{7,8}$ However, there is no specific treatment for AKU osteoarthritis. Management is usually conservative, largely based on analgesia and arthroplasty, but replacement surgery may be offered for severely affected major joints.

Bone cell activity is regulated by two key pathways that are represented by the axis receptor activator of NF- $\mathrm{BB}$ (RANK)/ RANK ligand (RANKL)/osteoprotegerin (OPG) and Wnt/ $\beta$-catenin signaling. ${ }^{9}$ The former regulates osteoclast (OC) formation, and the latter regulates osteoblast differentiation. RANKL promotes differentiation and fusion of the OC precursors (OCPs) and activates mature OCs to resorb bone by binding to their specific receptor, RANK. ${ }^{10} \mathrm{OPG}$ competes with RANK in binding to RANKL, preventing its pro-osteoclastogenic effect. ${ }^{10}$ RANKL and OPG levels are modulated by canonical WNT pathway antagonists, such as Dickkopf-1 (DKK1) and sclerostin, which are key regulators of bone mass. ${ }^{11}$ The role of DKK1 and sclerostin in several bone diseases has been established. ${ }^{9,12,13}$ In particular, DKK1 inhibits the maturation of osteoblasts, decreases OPG levels, and increases RANKL expression in osteoblasts, thereby shifting the OPG/RANKL ratio in favor of bone resorption. ${ }^{14}$

Our aim was to deepen the pathophysiology of bone disease in AKU patients, focusing our attention on the measurement of RANKL, OPG, sclerostin, and DKK1 serum levels and on the study of the mechanism/s regulating osteoclastogenesis. The results arising from our study will suggest new therapeutic strategies aimed to improve bone health in AKU patients.

\section{Materials and Methods}

\section{Patient Data and Procedures}

Nine patients (eight males and one female), including two children (aged 8.5 and 13.2 years) and seven adults (mean age, $31.7 \pm 15.9$ years), followed up at our Hospital Unit for AKU, were enrolled in the study. Age at diagnosis ranged between the neonatal period and 54 years. There were four couples of siblings and one unrelated patient. All patients were given a diet of unrestricted protein intake and were treated with vitamin $\mathrm{C}$ by oral administration. Diagnosis of AKU was established on the basis of the darkening of urine on standing to air and confirmed by the 24-hour urinary excretion of HGA $>18$ mmol, as measured by high-performance liquid chromatography. ${ }^{15}$ The diagnosis of ochronosis was based on the bluish- black pigmentation of the sclerae and of the cartilages of the external ears and of the nose, together with the radiological evidence of extensive spine involvement with degeneration and calcification of intervertebral disks. The adult patients had normal working and relational activities, and none had significant invalidities. None took treatment affecting bone metabolism.

A control group of 16 pediatric subjects (seven males and nine females; mean age, $7.2 \pm 3.1$ years) was recruited. It comprised children with the same age range of the AKU patients and not taking any medications, referred to our hospital for minor surgery or electrocardiographic screening, and adults recruited on a voluntary basis. As a control group for adult AKU patients, we enrolled 15 (nine males and six females; mean age, $32.7 \pm 17.9$ years) adult healthy volunteers.

Informed consent was obtained from all patients included in the study. Ethic committee permission was obtained before starting the recruitment. Personal information and all of the data relating to the patients were treated according to the local data protection policy.

All patients underwent physical examination, including general body and neurologic assessment and evaluation of joint mobility and pain, gait, and skeletal abnormalities. Ochronotic spondyloarthropathy was assessed using the simplified radiological staging by Jebaraj et al, ${ }^{16}$ based on radiographic findings of the thoracolumbar spine. The severity of disease was also assessed using the AKU Severity Score Index. ${ }^{17}$

\section{Biochemical Assessments}

Tyrosine metabolite production was assessed through measurement of HGA by gas chromatography, and results were expressed in $\mathrm{mmol} / \mathrm{mol}$ creatinine. $25(\mathrm{OH})$-vitamin D serum concentration was measured by immunologic tests based on the principle of chemiluminescence (Liaison assay; DiaSorin, Stillwater, MN). Osteocalcin serum concentration was measured by Enzyme Immuno Assay, using a commercial kit (IBL International, Hamburg, Germany). Calcium, phosphorus, and alkaline phosphatase serum concentrations were measured by nephelometric method. RANKL, OPG, sclerostin, and DKK1 were measured as previously reported. ${ }^{18,19}$ Calcitonin and C-terminal telopeptide of collagen type 1 (CTX; INCBiomedica, Vienna, Austria) were measured in the sera, using commercially available enzyme-linked immunosorbent assay kits (Cell Biolabs, San Diego, CA), according to the manufacturer's instructions. The absorption was determined with an enzyme-linked immunosorbent assay reader (Eon; BioTek, Swindon, UK). The intra-assay and interassay $\mathrm{CV}$ was $<5 \%$ for all assays. Urine calcium/creatinine ratio and glomerular filtration rate were measured in urine collected after a 12-hour overnight fast.

\section{Bone Mineral Measurements}

Bone mineral density (BMD) was measured by dual-energy X-ray absorptiometry (Hologic QDR 2000; Hologic, 
Table 1 Severity Score Index of Patients (AKUSSI)

\begin{tabular}{lcclc}
\hline $\begin{array}{l}\text { Patient } \\
\text { no. }\end{array}$ & $\begin{array}{l}\text { Clinical } \\
\text { score }\end{array}$ & $\begin{array}{l}\text { Spine } \\
\text { score }\end{array}$ & $\begin{array}{l}\text { Nonspine } \\
\text { score }\end{array}$ & $\begin{array}{l}\text { Total } \\
\text { AKUSSI score }\end{array}$ \\
\hline 1 & 0 & 0 & 0 & 0 \\
2 & 8 & 0 & 0 & 8 \\
3 & 0 & 4 & 0 & 4 \\
4 & 4 & 4 & 2 & 10 \\
5 & 0 & 2 & 2 & 4 \\
6 & 8 & 2 & 0 & 10 \\
7 & 8 & 6 & 0 & 14 \\
8 & 40 & 6 & 6 & 52 \\
9 & 52 & 12 & 8 & 72 \\
\hline
\end{tabular}

AKUSSI, Alkaptonuria Severity Score Index.

Waltham, MA) at the lumbar spine (LS) in the anteroposterior projection and at femoral neck. The dual-energy X-ray absorptiometry device was calibrated daily by an anthropomorphic phantom, with a precision error of $<1.0 \%$ and of $2.6 \%$ for spine and femoral scans, respectively. The results are also expressed in terms of standard units (Z-score). According to the current recommendation of the International Osteoporosis Foundation and World Health Organization, the National Health and Nutrition Examination Survey reference database was used as a reference range for BMD comparison. ${ }^{20}$

\section{Cells and Culture Conditions}

OCs were obtained from peripheral blood mononuclear cells (PBMCs) of AKU subjects and controls. PBMCs were isolated by centrifugation of peripheral blood samples over Histopaque 1077 density gradient (Sigma Chemical, St. Louis, MO). They were diluted at $1 \times 10^{6}$ cells $/ \mathrm{mL}$ in $\alpha$-minimal essential medium (Invitrogen, Paisley, UK) and supplemented with $10 \%$ fetal bovine serum, $100 \mathrm{IU} / \mathrm{mL}$ penicillin, and $100 \mu \mathrm{g} / \mathrm{mL}$ streptomycin (Life Technologies, Inc. Ltd, Uxbridge, UK). To obtain fully differentiated human OCs, the PBMCs were then cultured for approximately 20 days in the presence or absence of $25 \mathrm{ng} / \mathrm{mL}$ recombinant human macrophage colonystimulating factor and $30 \mathrm{ng} / \mathrm{mL}$ RANKL (R\&D Systems, Minneapolis, MN). In some experiments, PBMCs were cultured in the presence of $100 \mathrm{ng} / \mathrm{mL}$ RANK-constant fragment (R\&D Systems), $10 \mathrm{ng} / \mathrm{mL}$ tumor necrosis factor (TNF)receptor 1 -constant fragment, or $100 \mathrm{ng} / \mathrm{mL}$ anti-LIGHT (homologous to lymphotoxins exhibiting inducible expression and competing with herpes simplex virus glycoprotein $\mathrm{D}$ for herpes virus entry mediator, a receptor expressed by $\mathrm{T}$ lymphocytes) monoclonal antibody (R\&D Systems).

At the end of the culture period, mature OCs were identified as tartrate-resistant acid phosphatase-positive multinucleated cells (Sigma Aldrich, Milan, Italy) containing three or more nuclei. OC resorbing activity was evaluated by plating the PBMCs on Millennium multiwell slides (Millennium Biologix, Kingston, ON, Canada). To observe and measure the pits, the cells were eliminated by washing each well with $\mathrm{NaOCl}$. The photomicrographs were obtained using an
Ellipse E400 microscope (Nikon, Tokyo, Japan) equipped with Nikon Plan Fluor $10 \times / 0.30$ differential interference contrast line. The microscope was connected with a Nikon digital camera DxM 1200; the acquisition software was Lucia G version 4.61 (build 0.64 ) from Nikon Italy (Florence, Italy).

\section{Flow Cytometry Analysis}

Fresh peripheral blood samples from subjects with AKU and controls were stained with suitable conjugated antibody to characterize OCPs as Pc5-CD14, phosphatidylethanolamine$\mathrm{CD} 51 / 61$, and fluorescein isothiocyanate-CD11b (all Beckmann Coulter, Milan, Italy). Cells were incubated for 30 minutes at room temperature, and events were acquired using an FC500 (Beckmann Coulter) flow cytometer. TNF- $\alpha$-positive cells were stained using TNF- $\alpha$-phosphatidylethanolamine secretion kit, according to the manufacturer's instruction (Miltenyi Biotec S.r.l., Bologna, Italy), and further characterized using Pc5-CD14. The area of positivity was determined using an isotype-matched monoclonal antibody, and a total of $10^{6}$ events for each sample were acquired.

\section{RNA Isolation and Real-Time PCR Amplification}

Freshly isolated PBMCs of subjects with AKU and controls were subjected to mRNA extraction using spin columns (RNeasy; Qiagen, Hilden, Germany), according to the manufacturer's instructions. The extracted RNA was reverse transcribed using iScript Reverse Transcription Supermix (Bio-Rad Laboratories, Hercules, CA). cDNA was amplified with the SsoFast EvaGreen Supermix (Bio-Rad Laboratories), and the PCR amplification was performed using the Chromo4 Real-Time PCR Detection System (Bio-Rad Laboratories), as previously reported. ${ }^{21}$ The following primer pairs were used for the real-time PCR amplification: RANKL, 5'-CGT-TGGATCACAGCACAT-3' (sense) and $5^{\prime}$-GCTCCTCTTGGCCAGTC-3' (antisense); TNF- $\alpha, 5^{\prime}$ ATCTACTCCCAGGTCCTC-3' (sense) and 5'-GATGCGGCTGATGGTGT-3' (antisense); RANK, 5'-CAGGATGCTCTCATTGGTCAG-3' (sense) and 5'-AGAAAGGAGGTGTGGATTGC-3' (antisense); colony-stimulating factor-1 receptor, 5'-GTGGCAGGAAGGTGATGTC- $3^{\prime}$ (sense) and 5'-GCTTGGTGTT-GTTGTGTTGG-3' (antisense); LIGHT, $5^{\prime}$-CAGTGTTTG-TGGTGGATGG-3' (sense) and 5'-GGG TTGACCTCGTGAGAC-3' (antisense); and GAPDH, 5'TCATCCCTGCC-TCTACTG- $3^{\prime}$ (sense) and 5'-TGCTTCA CCACCTTCTTG-3' (antisense).

\section{Statistical Analysis}

For statistical analysis, the Statistical Package for the Social Sciences for Windows version 22.0 (SPSS Inc., Chicago, IL) was used. Comparison between groups was performed by $t$-test. Correlations were analyzed with Spearman correlation test. The limit of statistical significance was set at $P=0.05$. 
Table 2 Urine Calcium/Creatinine Ratio, Calcitonin, and GFR

\begin{tabular}{llll}
\hline Patient & Calcitonin, & $\begin{array}{l}\text { Calciuria/ } \\
\text { Creatinine ratio, } U\end{array}$ & $\begin{array}{l}\text { GFR, } \mathrm{mL} / \mathrm{min} \\
\text { per } 1.73 \mathrm{~m}^{2} \\
\text { (CKD-EPI formula) }\end{array}$ \\
\hline 1 & $\mathrm{pg} / \mathrm{mL}$ & 0.46 & 166 \\
2 & 1.89 & 0.10 & 136 \\
3 & $<1$ & 0.25 & 133 \\
4 & $<1$ & 0.15 & 124 \\
5 & $<1$ & 1.1 & 132 \\
6 & $<1$ & 0.79 & 137 \\
7 & 3.24 & 0.29 & 129 \\
8 & 9.37 & 0.31 & 109 \\
9 & 9.3 & 0.33 & 98 \\
\hline
\end{tabular}

Reference ranges: calcitonin, 1 to $11.8 \mathrm{pg} / \mathrm{mL}$; calciuria/creatinine ratio, aged 5 to 14 years, $<0.21$; and adults, $<0.33$; and GFR, $>90 \mathrm{~mL} / \mathrm{min}$ per $1.73 \mathrm{~m}^{2}$.

CKD-EPI, Chronic Kidney Disease Epidemiology Collaboration; GFR, glomerular filtration rate.

\section{Results}

\section{Baseline Characteristics}

Results of the AKU Severity Score Index are reported in Table 1. Physical examination highlighted ochronosis in four of nine patients (age at onset, $\geq 28.5$ years). Target organ damage with aortic stenosis and mild mitralic and/or tricuspidalic regurgitation was diagnosed by echocardiography in three subjects (33\%). Renal stones were found in two patients, and prostatic stones were found in one of the investigated subjects. Slight skeletal abnormalities were detected in most subjects (six of nine), and were treated with conservative procedures in all cases. Three patients had undergone surgery: one for bone fractures and the other two (siblings) for arthroplasty. Only one patient, the oldest one, manifested pain at joint mobilization.

The results of calciuria/creatinine ratio, calcitonin, and glomerular filtration rate, expressed in terms of standardized values, of the study groups are shown in Table 2. Cases 1, 5, and 6 displayed high fasting urine calcium/creatinine ratio with respect to the reference range. Otherwise, calcitonin levels and glomerular filtration rate were in the normal ranges for all patients. Densitometric data and bone metabolism markers of the study population are reported in Table 3. In the two pediatric AKU patients, LS-BMD and femoral-BMD Z-scores were within the normal range, but were reduced with respect to the control subjects. Otherwise, in the adult AKU patients, LS-BMD T-scores were inside the normal range, but femoral-BMD T-score reached osteopenic levels. In particular, two of the seven adult patients (the youngest ones) showed femoral-BMD T-scores between 0 and -1 ; five patients (71.4\%) had femoral-BMD $\mathrm{Z}$-scores between -1 and -2.5 . Interestingly, the levels of the resorption marker CTX were reduced in pediatric AKU, but increased in adults with respect to controls $(P<0.004)$. $25(\mathrm{OH})$-vitamin $\mathrm{D}$ levels were reduced in adult patients compared with controls $(P<0.04)$. The obtained results prompted us to evaluate serum levels of cytokines involved in osteoclastogenesis (RANKL and OPG) or in osteoblastogenesis (DKK1 and sclerostin).

\section{High Serum Levels of RANKL in Adult AKU Patients}

RANKL serum levels significantly increased in adult AKU patients compared with controls $(P<0.01)$; conversely, OPG levels were reduced in both pediatric and adult AKU patients with respect to controls $(P<0.05)$, thus shifting the

Table 3 Densitometric Data and Markers of Bone Metabolism

\begin{tabular}{lccccc}
\hline Variable & $\begin{array}{l}\text { Pediatric } \\
\text { AKU1 }\end{array}$ & $\begin{array}{c}\text { Pediatric } \\
\text { AKU2 }\end{array}$ & $\begin{array}{c}\text { Pediatric controls } \\
(n=16)\end{array}$ & $\begin{array}{c}\text { Adult AKU } \\
(n=7)\end{array}$ & $\begin{array}{c}\text { Adult controls } \\
(n=15)\end{array}$ \\
\hline LS-BMD Z-score & -0.9 & -0.3 & $0.13 \pm 0.80$ & NA & NA \\
LS-BMD T-score & NA & NA & NA & $-0.76 \pm 0.86$ & NA \\
Femoral-BMD Z-score & -0.3 & -0.1 & $0.1 \pm 0.3$ & NA & NA \\
Femoral-BMD T-score & NA & NA & NA & $-1.57 \pm 0.64$ & $21.4 \pm 2.62^{*}$ \\
25(OH)-vitamin D, ng/mL & 17.2 & 33.6 & $38.6 \pm 14.7$ & $21.4 \pm 2.62$ & $34.2 \pm 3.8$ \\
Osteocalcin, ng/mL & 51.5 & 21.9 & $38.3 \pm 19.2$ & $31.44 \pm 12.51$ & $38.5 \pm \pm 3.12$ \\
PTH, pg/mL & 23.7 & 13.3 & $19.25 \pm 5.1$ & $9.35 \pm 0.45$ & $10.11 \pm 0.60$ \\
Ca, mg/dL & 9.2 & 10.1 & $9.71 \pm 0.40$ & $2.95 \pm 0.38^{*}$ & $4.60 \pm 0.22$ \\
P, mg/dL & 4.2 & 3.36 & $4.54 \pm 1.40$ & $56.8 \pm 7.56$ & $74.6 \pm 23$ \\
ALP, U/L & 158 & 61 & $199.6 \pm 65.8$ & $0.47 \pm 0.11^{* *}$ & $0.28 \pm 0.110$ \\
CTX, ng/mL & 0.991 & 0.457 & $1.27 \pm 0.49$ & $82.5 \pm 33.64^{* *}$ & $50.9 \pm 21.8$ \\
RANKL, pg/mL & 52.31 & 71.9 & $87.2 \pm 17.8$ & $387.6 \pm 100.5^{*}$ & $531.1 \pm 109$ \\
OPG, pg/mL & 487.55 & 309.46 & $436.7 \pm 95.5$ & $2130 \pm 1001$ & $1995 \pm 701$ \\
Sclerostin, pg/mL & 1500 & 1195 & $1365 \pm 267$ & $3174 \pm 303$ & $3336 \pm 675$ \\
DKK-1, pg/mL & 3590 & 2601 & $3521 \pm 1173$ &
\end{tabular}

Data are expressed as means \pm SD.

${ }^{*} P<0.05,{ }^{* *} P<0.01$ versus adult controls.

AKU, alkaptonuria; ALP, alkaline phosphatase; BMD, bone mineral density; Ca, calcium; CTX, C-terminal telopeptide of type I collagen; DKK-1, dickkopf-1; LS, lumbar spine; NA, not available; OPG, osteoprotegerin; P, phosphorus; PTH, parathyroid hormone; RANKL, receptor activator of NF- $\mathrm{B}$ ligand. 


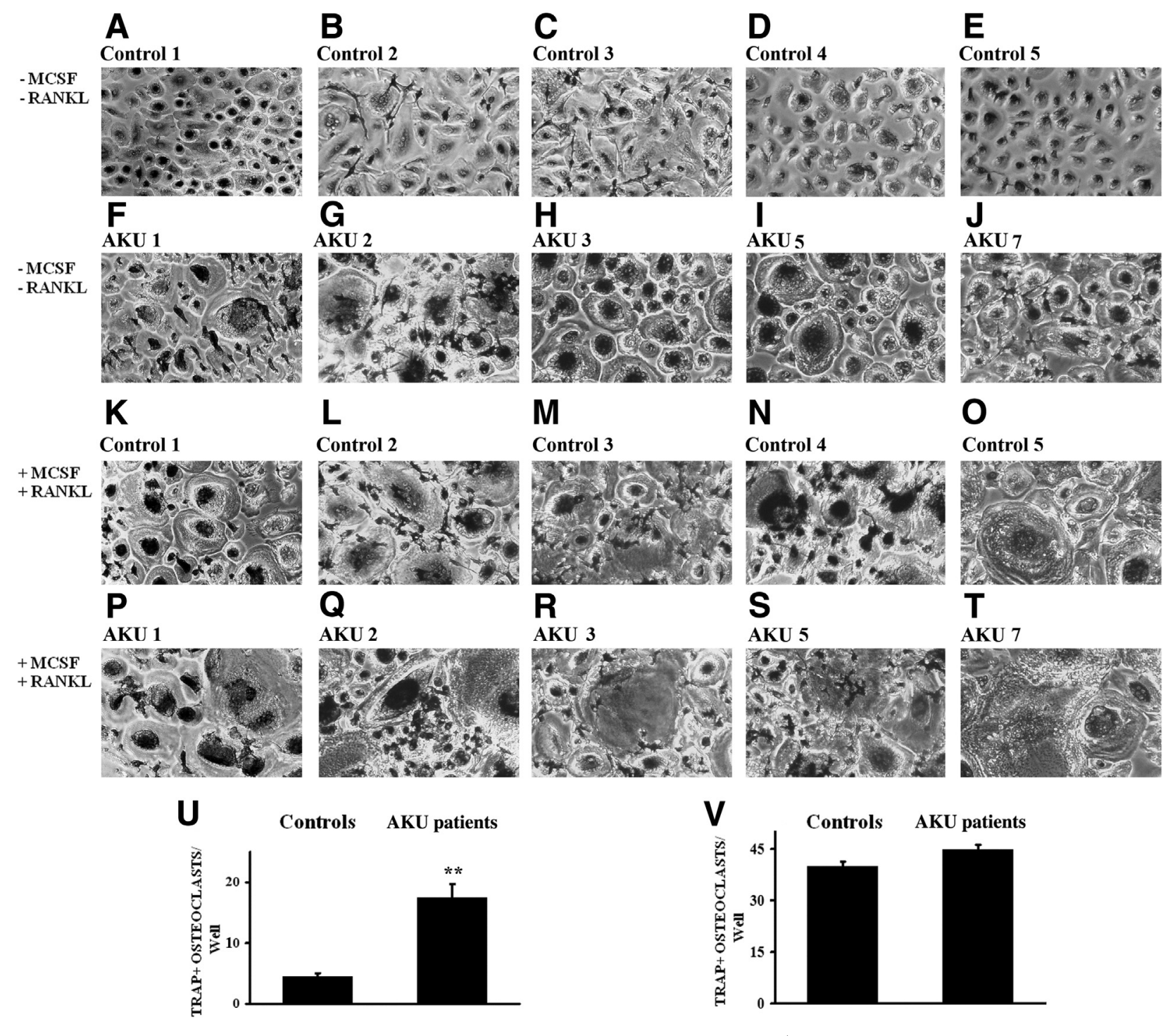

Figure 1 Osteoclast formation in AKU patients. Tartrate-resistant acid phosphatase-positive $\left(\operatorname{TRAP}^{+}\right)$and multinucleated cells with three or more nuclei, identified as osteoclasts (OCS), were obtained under different culture conditions from peripheral blood mononuclear cells (PBMCs) of controls and AKU patients. A-E: Few small TRAP ${ }^{+}$and multinucleated cells are detected in unstimulated PBMC cultures from five representative controls. F-J: Spontaneous osteoclastogenesis can be observed in unstimulated PBMCs from five representative AKU patients. $\mathbf{K}-\mathbf{T}$ : In parallel, PBMCs from controls (K-0) and AKU patients $(\mathbf{P}-\mathbf{T})$ were stimulated with macrophage colony-stimulating factor (MCSF) and RANKL, leading to the formation of multinucleated TRAP ${ }^{+}$cells. $\mathbf{U}$ and V: The OC counts arising for the unstimulated (U) or stimulated (V) PBMC cultures performed from all controls and patients in quintuplicate. Data are expressed as means $\pm \mathrm{SEM}(\mathbf{U}$ and $\mathbf{V}) .{ }^{* *} P<0.01$ versus control. Original magnification, $\times 200(\mathbf{A}-\mathbf{T})$.

RANKL/OPG ratio in favor of RANKL and, therefore, of bone resorption. Sclerostin and DKK1 levels were not significantly altered in AKU subjects compared with controls. In adults, with adjustment for age, the increased serum levels of RANKL significantly correlated with the following: OPG $(r=-0.903, P<0.0001)$, CTX $(r=0.291, \quad P<0.0001)$, femoral-BMD T-score $(r=-0.275, P<0.0001)$, LS-BMD Z-score $(r=0.785$, $P<0.0001), 25(\mathrm{OH})$-vitamin D $(r=-0.388, P<0.0001)$, calcium $(r=0.170, P<0.008)$, phosphorus $(r=-0.341$, $P<0.0001)$, and alkaline phosphatase $(r=-0.241$, $P<0.0001)$. Otherwise, the reduced serum levels of OPG correlated with CTX $(r=-0.443, P<0.0001)$, osteocalcin $(r=-0.566, P<0.0001)$, calcium $(r=-0.394$, $P<0.0001)$, and phosphorus $(r=0.183, P<0.009)$. CTX serum levels in adults significantly correlated with femoralBMD T-score $(r=-0.793, P<0.0001)$ and LS-BMD T-score $(r=0.684, P<0.0001)$.

The obtained results prompted us to evaluate osteoclastogenesis in AKU patients.

\section{Spontaneous Osteoclastogenesis in Unstimulated Cultures of PBMCs from AKU Patients}

Few multinucleated (nuclei, $>3$ ) tartrate-resistant acid phosphatase-positive OCs were identified in the 


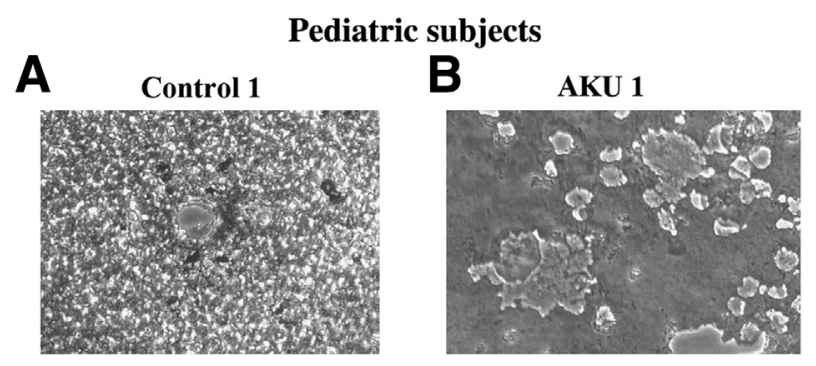

Adult subjects
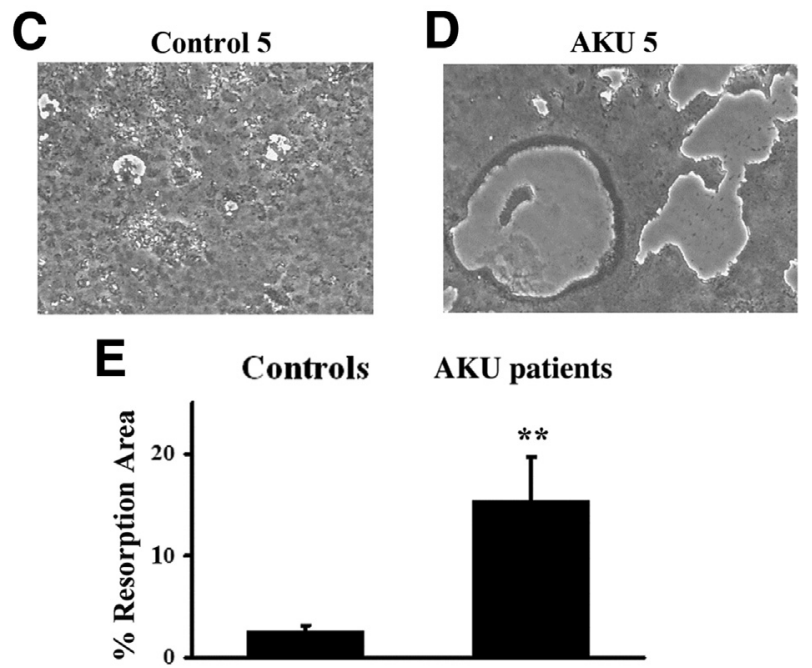

Figure 2 A-D: Photomicrographs of the resorption area eroded on Millennium 0steologic slides by the osteoclasts derived from unstimulated peripheral blood mononuclear cell cultures of representative pediatric control 1 (A) and AKU patient 1 (B) as well as from representative adult control 5 (C) and AKU patient 5 (D). E: The percentage of the resorbed area was quantified using an image analyzer and reported. Data are expressed as means \pm SEM (E). $n=6$ controls ( 3 pediatric +3 adult subjects; $E$ ); $n=9$ patients $(\mathbf{E})$. $* * P<0.01$ versus control. Original magnification, $\times 200$ (A-D).

unstimulated PBMC cultures from five controls (OC average number/well, $5 \pm 4$ ) (Figure 1, A-E), whereas a significant number were observed in the cultures from $\mathrm{AKU}$ patients (OC average number/well, $20 \pm 4$ ) (Figure 1, F-J and $\mathrm{U})$. Moreover, the addition of macrophage colonystimulating factor and RANKL was essential to promote osteoclastogenesis in controls' cultures (OC average number/well, $45 \pm 7$ ) (Figure 1, K-O). The addition of the same cytokines to the cultures of AKU patients significantly increased the OC number (OC average number/well, $39 \pm 6 ; P<0.01$ ) (Figure $1, \mathrm{P}-\mathrm{T}$ and $\mathrm{V}$ ). The OCs became bigger compared with those in the parallel unstimulated cultures (Figure 1, F-J). Moreover, fewer and smaller resorption areas were found in unstimulated PBMC cultures from controls (Figure 2, A-C), compared with AKU patients (Figure 2, B-D), as quantified in Figure 2E.

Significant correlations were found for adults between osteoclastogenic potential and serum levels of the following: RANKL $(r=0.200, P<0.01)$, DKK1 $(r=-0.924$, $P<0.0001)$, sclerostin $(r=0.336, P<0.0001)$, CTX $(r=0.612, P<0.0001), 25(\mathrm{OH})$-vitamin $\mathrm{D}(r=-0.293$, $P<0.0001)$, osteocalcin $(r=-0.340, P<0.0001)$, calcium $(r=0.154, P<0.05)$, alkaline phosphatase $(r=-0.971$, $P<0.0001)$, phosphorus $(r=-0.412, P<0.0001)$, femoralBMD Z-score $(r=0.732, P<0.0001)$, and LS-BMD $\mathrm{Z}$-score $(r=-0.684, P<0.0001)$. To understand the mechanism underlying the spontaneous OC formation, we evaluated the percentage of circulating OCPs and the expression of pro-osteoclastogenic factor by circulating cells.

\section{Increased Circulating 0steoclast Precursors and Pro- Osteoclastogenic Cytokines in AKU Patients}

The expression of circulating OCP surface markers, $\mathrm{CD} 14^{+}$, $\mathrm{CD} 51 / 61^{+}$, and $\mathrm{CD} 11 \mathrm{~b}^{+}$, showed statistically significant changes between subjects with AKU and controls (Figure 3). In addition, by real-time PCR, it was found that in AKU patients the monocytes expressed higher levels of RANK (Figure 4A), receptor of RANKL, compared with controls, thus supporting the spontaneous OC formation. Furthermore, lymphomonocytes also expressed high levels of RANKL (Figure 4B), together with LIGHT (Figure 4C) and TNF- $\alpha$ (Figure 4D), at the mRNA level. Flow cytometric analysis also supported the lower expression of RANKL on controls' lymphomonocyte (Figure 5A) with respect to AKU patients (Figure 5, B and C), as quantified in
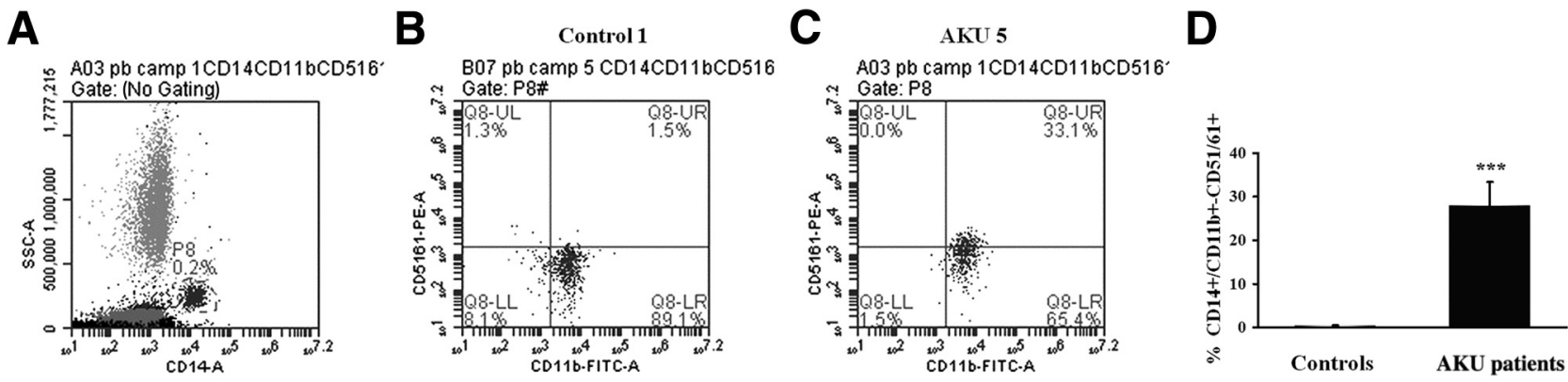

Figure 3 0steoclast precursors in AKU patients. A: $\mathrm{CD}_{1} 4^{+}$monocytes were gated using a specific $\mathrm{CD} 14-\mathrm{PerCP}$ antibody to derive the study of $\mathrm{CD} 11 \mathrm{~b}^{+} /$ CD51/61+ cells. B and C: A representative flow cytometry dot plot shows the percentage of circulating osteoclast precursors (OCPs), identified as CD14 ${ }^{+} /$

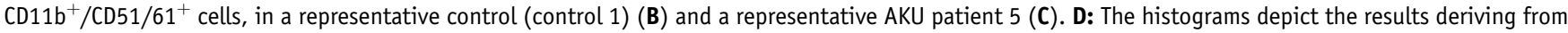

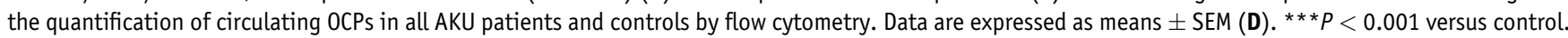
FITC, fluorescein isothiocyanate; PE, phosphatidylethanolamine; SSC, side scatter. 

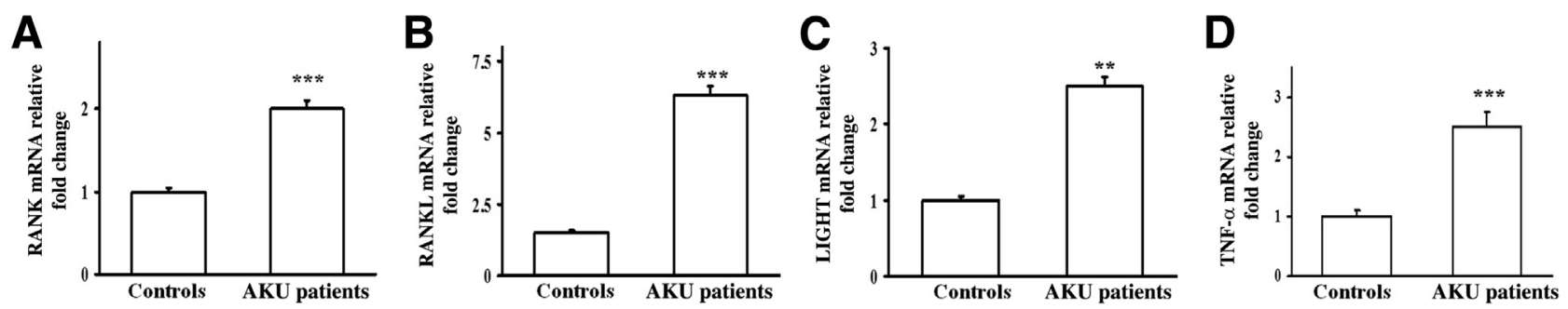

Figure 4 RANK, RANKL, LIGHT, and tumor necrosis factor (TNF)- $\alpha$ expression on circulating cells from AKU patients. mRNA levels of RANK by monocytes (A) as well as of RANKL (B), LIGHT (C), and TNF- $\alpha$ (D) by lymphomonocytes from all controls and AKU patients are reported. Data are expressed as means \pm SEM (A-D). ${ }^{*} P<<0.01,{ }^{* * *} P<0.001$ versus control.

Figure 5D. By flow cytometry, a smaller percentage of monocytes expressing TNF- $\alpha$ in control samples compared with AKU patients were also demonstrated (Figure 5, E-G). TNF- $\alpha$ and LIGHT are known to amplify the RANKL pro-osteoclastogenic effect in bone diseases. ${ }^{22,23}$ Consistently, a dose-dependent inhibitory effect exerted by RANK-Fc and anti-LIGHT on osteoclastogenesis in AKU PBMC cultures was demonstrated (Figure 5H).

\section{Discussion}

The results of the present study highlighted the elevated levels of RANKL and CTX and the reduced amounts of OPG in the sera of AKU adult patients and the possible involvement of these cytokines in the altered OC activity associated with this disease. In the two pediatric subjects affected by AKU, the serum levels of RANKL were lower with respect to controls, suggesting that the enhanced bone remodeling occurs with aging. Consistently with these results, the densitometric parameters were normal in the two AKU children, whereas they were altered in AKU adult subjects both at lumbar and femoral sites. Aliberti et $\mathrm{al}^{7}$ reported conflicting densitometric results because LS-BMD was normal or markedly increased, probably because of intervertebral disk calcification and osteophyte formation, whereas femoral neck BMD was almost always reduced. Consistent with the data, the same authors found increased N-telopeptides of type I collagen urinary excretion, suggesting that ochronosis is related with increased bone resorption by OCs in vivo. ${ }^{6}$ As also reported by Aliberti et al, ${ }^{7}$ some patients showed increased calciuria/ creatinine ratio, which suggests a negative bone balance.
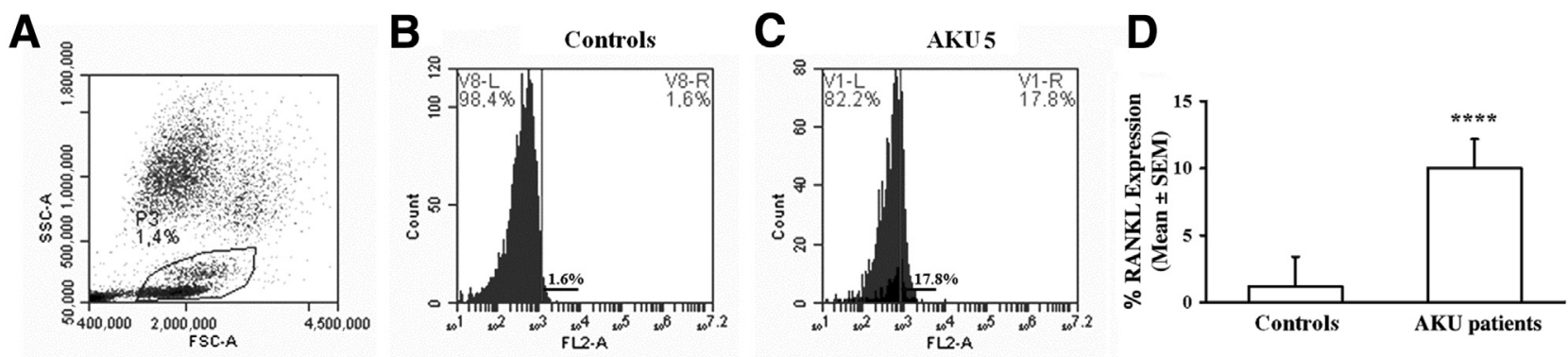

RANKL-PE
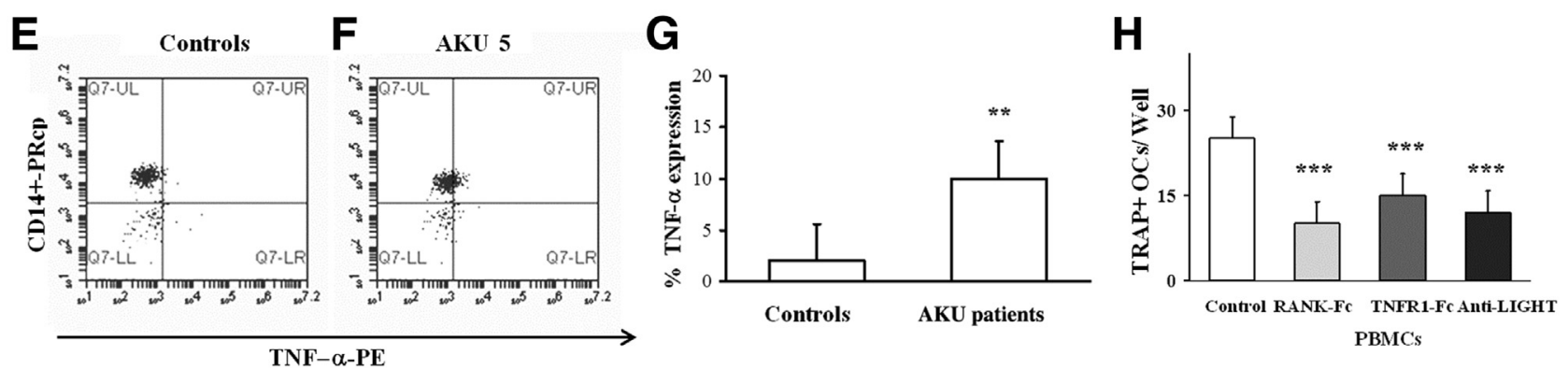

Figure 5 RANK, RANKL, LIGHT, and tumor necrosis factor (TNF)- $\alpha$ expression in AKU patients. A: RANKL expression was evaluated by gating on lymphomonocyte population. B-D: Flow cytometry analysis of RANKL on peripheral blood mononuclear cells (PBMCs) from a representative control (1; B) and AKU patient $(5 ; \mathbf{C})$, together with the histograms showing the results arising from the quantification of RANKL levels in all patients and controls (D). E and F: Flow cytometry dot plots showing TNF- $\alpha$ expression on $\mathrm{CD}_{14}{ }^{+}$monocytes, from a representative control 5 (E) and a representative AKU patient 5 (F). G: The histogram reports the results arising from the evaluation of TNF- $\alpha$ levels in all patients and controls by flow cytometry. H: The formation of multinucleated tartrate-resistant acid phosphatase-positive (TRAP ${ }^{+}$) osteoclasts $(0 \mathrm{Cs})$ was measured in unstimulated PBMCs from all AKU patients cultured in the absence or presence of $100 \mathrm{ng} / \mathrm{mL}$ RANK-Fc and/or $10 \mathrm{ng} / \mathrm{mL}$ TNF-R1-Fc or $100 \mathrm{ng} / \mathrm{mL}$ anti-LIGHT. Data are expressed as means \pm SEM (D, G, and H). ${ }^{* * P}<0.01$, $* * * P<0.001$, and ${ }^{* * * * P}<0.0001$ versus control. FSC, forward scatter; PRcp, peridinin-chlorophyll-protein complex; SSC, side scatter. 
Genovese et $\mathrm{al}^{8}$ also found high levels of CTX in AKU patients and, in accordance with presented data, the same authors highlighted unchanged osteocalcin levels compared with controls, suggesting that bone impairment in AKU did not affect osteoblasts. These findings may explain the similar levels of DKK1 and sclerostin that we found in the sera from patients and controls. Thus, these results demonstrated that bone impairment in AKU may be strictly attributable to high OC activity. RANKL and OPG have been suggested to be the critical factors in determining OC activation at the bone level, with higher serum RANKL/OPG ratios being a marker for up-regulation of osteoclastogenesis. ${ }^{17,21,24-26}$ Alterations in RANKL and OPG amounts have been demonstrated in several bone loss-associated diseases, including arthritis. ${ }^{27}$ Interestingly, it was also found that lymphomonocytes from AKU patients expressed high amounts of RANKL, thus supporting the high osteoclastogenic potential of PBMCs from these patients. In fact, the neutralization of RANKL through RANK-Fc inhibited osteoclastogenesis. In addition, in AKU patients, the high osteoclastogenesis potential of PBMCs is sustained by the elevated percentage of circulating OCPs and by the high levels of LIGHT and TNF- $\alpha$. Consistently, the neutralization of both these cytokines inhibited spontaneous OC formation in vitro. LIGHT and TNF- $\alpha$ are known to potentiate RANKL's effect on osteoclastogenesis in bone diseases and with particular remark in erosive arthritis. $^{22,23,28}$ The obtained results suggested primarily RANKL, but also TNF- $\alpha$ and LIGHT, as new therapeutic target for AKU management. However, it cannot be excluded that osteoblastic cells, as the main source of RANKL and OPG, from AKU patients may contribute to the alteration of their levels; however, this possibility was not explored. Currently, there is no successful therapy for AKU patients, and the management of this pathology is palliative and includes physiotherapy, pain control, and joint replacement surgery. Recent studies clearly pointed out that oxidative stress is one of the most important factors in AKU pathogenetic cascade. ${ }^{29-32}$ It was demonstrated that HGA undergoes spontaneous oxidation into 1,4-benzoquinone-2-acetic acid and that this conversion is concomitant with the production of oxygen radicals, responsible for the alterations of connective tissues observed in $\mathrm{AKU} .^{33}$ Our patients are treated with vitamin $\mathrm{C}$, an antioxidant able to reduce the conversion of HGA to 1,4-benzoquinone-2-acetic acid through oxidation. However, Sealock et $\mathrm{al}^{34}$ showed that although vitamin $\mathrm{C}$ reduced the HGA-to-1,4-benzoquinone-2-acetic acid conversion, it did not influence HGA urinary excretion. In addition, vitamin $\mathrm{C}$ in the presence of HGA can induce a marked increase of carbonylated proteins, having an ultimate pro-oxidant effect. ${ }^{35}$

During the past years, emphasis has been given to the use of nitisinone in the management of this disorder. ${ }^{36}$ Nitisinone is a triketone herbicide that inhibits 4-hydroxyphenylpyruvate dioxygenase, an enzyme involved in the conversion of hydroxyphenylpyruvate to HGA. It significantly decreases urinary excretion of HGA in a dose-dependent manner, and it was well tolerated in a recent trial including 40 patients, within a study time of 4 weeks. ${ }^{37}$ It is currently used for the treatment of hereditary tyrosinemia type I, but its clinical efficacy in AKU is under investigation (Suitability of Nitisinone in Alkaptonuria 2; http://www.ClinicalTrials.gov, last accessed October 17, 2017). In particular, data are needed to evaluate the most effective timing in the progression of the disease, for initiating nitisinone, to prevent massive pigment deposition, and to determine if other compounds can be used with the same purpose and fewer adverse effects. $^{38,39}$

Recently, in the management of arthritis, inhibitors of key pro-osteoclastogenic molecules are giving promising results ${ }^{11}$ which may be extended to the bone disease associated with AKU. ${ }^{40}$ Denosumab is a fully human monoclonal antibody against RANKL approved for the treatment of postmenopausal women with osteoporosis at increased (United States) or high risk (European Union) of fracture. During the pivotal 3-year fracture trial [Fracture REduction Evaluation of Denosumab in Osteoporosis every 6 Months (FREEDOM)], 60 mg denosumab (Prolia; Amgen Inc., Thousand Oaks, CA) given subcutaneously every 6 months significantly reduced bone turnover markers, increased BMD, and reduced new vertebral (68\%), hip (40\%), and nonvertebral $(20 \%)$ fractures compared with placebo in postmenopausal women with osteoporosis. ${ }^{41}$ Thus, the high levels of RANKL in adult AKU patients, together with osteopenia, suggest the potential use of denosumab in this cohort.

In conclusion, our results demonstrate that AKU adults have high levels of RANKL, and the spontaneous osteoclastogenesis as well as the high percentage of OCPs are already evident in children. Our findings suggest that, although the macroscopic injury becomes evident in adulthood, the bone remodeling is already altered in children/ young AKU patients; thus, we propose the importance of a preventive approach in these patients, in agreement with previous studies. ${ }^{41}$ Also, neutralizing RANKL in AKU patients may represent a new pharmacologic approach in the management of this pathology.

\section{Acknowledgment}

We thank Bellocci Pasqua for technical support.

\section{References}

1. Scriver CR: The hyperphenylalaninemias and alkaptonuria. Edited by Goldman L, Bennett JC. In Cecil Textbook of Medicine. ed 21. Philadelphia: WB Saunders, 2000. pp. 1109-1110

2. Taylor AM, Wlodarski B, Prior IA, Wilson PJ, Jarvis JC, Ranganath LR, Gallagher JA: Ultrastructural examination of tissue in a patient with alkaptonuric arthropathy reveals a distinct pattern of binding of ochronotic pigment. Rheumatology 2010, 49:1412-1414

3. Millucci L, Spreafico A, Tinti L, Braconi D, Ghezzi L, Paccagnini E, Bernardini G, Amato L, Laschi M, Selvi E, Galeazzi M, Mannoni A, Benucci M, Lupetti P, Chellini F, Orlandini M, Santucci A: 
Alkaptonuria is a novel human secondary amyloidogenic disease. Biochim Biophys Acta 2012, 1822:1682-1691

4. Millucci L, Braconi D, Bernardini G, Lupetti P, Rovensky J, Ranganath L, Santucci A: Amyloidosis in alkaptonuria. J Inherit Metab Dis 2015, 38:797-805

5. Braconi D, Millucci L, Bernini A, Spiga O, Lupetti P, Marzocchi B, Niccolai N, Bernardini G, Santucci A: Homogentisic acid induces aggregation and fibrillation of amyloidogenic proteins. Biochim Biophys Acta 2017, 1861:135-146

6. Davison AS, Milan AM, Gallagher JA, Ranganath LR: Acute fatal metabolic complications in alkaptonuria. J Inherit Metab Dis 2016, 39: 203-210

7. Aliberti G, Pulignano I, Schiappoli A, Minisola S, Romagnoli E, Proietta M: Bone metabolism in ochronotic patients. J Intern Med 2003, 254:296-300

8. Genovese F, Siebuhr AS, Musa K, Gallagher JA, Milan AM, Karsdal MA, Rovensky J, Bay-Jensen AC, Ranganath LR: Investigating the robustness and diagnostic potential of extracellular matrix remodelling biomarkers in alkaptonuria. JIMD Rep 2015, 24:29-37

9. Baron R, Kneissel M: WNT signaling in bone homeostasis and disease: from human mutations to treatments. Nat Med 2013, 19:1791-1792

10. Mao B, Wu W, Davidson G, Marhold J, Li M, Mechler BM, Delius H, Hoppe D, Stannek P, Walter C, Glinka A, Niehrs C: Kremen proteins are Dickkopf receptors that regulate Wnt/beta-catenin signalling. Nature 2002, 417:664-667

11. Morvan F, Boulukos K, Clément-Lacroix P, Roman Roman S, SucRoyer I, Vayssière B, Ammann P, Martin P, Pinho S, Pognonec P, Mollat P, Niehrs C, Baron R, Rawadi G: Deletion of a single allele of the Dkk1 gene leads to an increase in bone formation and bone mass. J Bone Miner Res 2006, 21:934-945

12. Qiang YW, Chen Y, Stephens O, Brown N, Chen B, Epstein J, Barlogie B, Shaughnessy JD Jr: Myeloma-derived Dickkopf-1 disrupts Wnt-regulated osteoprotegerin and RANKL production by osteoblasts: a potential mechanism underlying osteolytic bone lesions in multiple myeloma. Blood 2008, 112:196-207

13. Dalbeth N, Pool B, Smith T, Callon KE, Lobo M, Taylor WJ, Jones PB, Cornish J, McQueen FM: Circulating mediators of bone remodeling in psoriatic arthritis: implications for disordered osteoclastogenesis and bone erosion. Arthritis Res Ther 2010, 12:R164

14. Diarra D, Stolina M, Polzer K, Zwerina J, Ominsky MS, Dwyer D, Korb A, Smolen J, Hoffmann M, Scheinecker C, van der Heide D, Landewe R, Lacey D, Richards WG, Schett G: Dickkopf-1 is a master regulator of joint remodeling. Nat Med 2007, 13:156-163

15. Bory C, Boulieu R, Chantin C, Mathieu M: Diagnosis of alkaptonuria: rapid analysis of homogentisic acid by HPLC. Clin Chim Acta 1990, $189: 7-12$

16. Jebaraj I, Chacko BR, Chiramel GK, Matthai T, Parameswaran A: A simplified staging system based on the radiological findings in different stages of ochronotic spondyloarthropathy. Indian J Radiol Imaging 2013, 23:101-105

17. Cox TF, Ranganath LR: A quantitative assessment of alkaptonuria: testing the reliability of two disease severity scoring systems. J Inherit Metab Dis 2011, 34:1153-1162

18. Brunetti G, Papadia F, Tummolo A, Fischetto R, Nicastro F, Piacente L, Ventura A, Mori G, Oranger A, Gigante I, Colucci S, Ciccarelli M, Grano M, Cavallo L, Delvecchio M, Faienza MF: Impaired bone remodeling in children with osteogenesis imperfecta treated and untreated with bisphosphonates: the role of DKK1, RANKL, and TNF- $\alpha$. Osteoporos Int 2016, 27:2355-2365

19. Giordano P, Brunetti G, Lassandro G, Notarangelo LD, Luciani M, Mura RM, Lazzareschi I, Santagostino E, Piacente L, Ventura A, Cavallo L, Grano M, Faienza MF: High serum sclerostin levels in children with haemophilia A. Br J Haematol 2016, 172:293-295

20. Kanis JA, Gluer CC: An update on the diagnosis and assessment of osteoporosis with densitometry. Committee of Scientific Advisors, International Osteoporosis Foundation. Osteoporos Int 2000, 11: 192-202
21. Faienza MF, Brunetti G, Ventura A, Piacente L, Messina MF, De Luca F, Ciccarelli M, Oranger A, Mori G, Natale MP, Gigante M, Ranieri E, Gesualdo L, Colucci S, Cavallo L, Grano M: Mechanisms of enhanced osteoclastogenesis in girls and young women with Turner's syndrome. Bone 2015, 81:228-236

22. Brunetti G, Rizzi R, Oranger A, Gigante I, Mori G, Taurino G, Mongelli T, Colaianni G, Di Benedetto A, Tamma R, Ingravallo G, Napoli A, Faienza MF, Mestice A, Curci P, Specchia G, Colucci S, Grano M: LIGHT/TNFSF14 increases osteoclastogenesis and decreases osteoblastogenesis in multiple myeloma-bone disease. Oncotarget 2014, 5:12950-12967

23. Lam J, Takeshita S, Barker JE, Kanagawa O, Ross FP, Teitelbaum SL: TNF-alpha induces osteoclastogenesis by direct stimulation of macrophages exposed to permissive levels of RANK ligand. J Clin Invest 2000, 106:1481-1488

24. Hofbauer LC, Khosla S, Dunstan CR, Lacey DL, Boyle WJ, Riggs BL: The roles of osteoprotegerin and osteoprotegerin ligand in the paracrine regulation of bone resorption. J Bone Miner Res 2000, $15: 2-12$

25. Bostanci N, Ilgenli T, Emingil G, Afacan B, Han B, Töz H, Atilla G, Hughes FJ, Belibasakis GN: Gingival crevicular fluid levels of RANKL and OPG in periodontal diseases: implications of their relative ratio. J Clin Periodontol 2007, 34:370-376

26. Faienza MF, Brunetti G, Colucci S, Piacente L, Ciccarelli M, Giordani L, Del Vecchio GC, D’Amore M, Albanese L, Cavallo L, Grano M: Osteoclastogenesis in children with 21-hydroxylase deficiency on long-term glucocorticoid therapy: the role of receptor activator of nuclear factor-kappaB ligand/osteoprotegerin imbalance. J Clin Endocrinol Metab 2009, 94:2269-2276

27. van Tuyl LH, Voskuyl AE, Boers M, Geusens P, Landewé RB, Dijkmans BA, Lems WF: Baseline RANKL:OPG ratio and markers of bone and cartilage degradation predict annual radiological progression over 11 years in rheumatoid arthritis. Ann Rheum Dis 2010, 69: $1623-1628$

28. Edwards JR, Sun SG, Locklin R, Shipman CM, Adamopoulos IE, Athanasou NA, Sabokbar A: LIGHT (TNFSF14), a novel mediator of bone resorption, is elevated in rheumatoid arthritis. Arthritis Rheum 2006, 54:1451-1462

29. Millucci L, Ghezzi L, Paccagnini E, Giorgetti G, Viti C, Braconi D, Laschi M, Geminiani M, Soldani P, Lupetti P, Orlandini M, Benvenuti C, Perfetto F, Spreafico A, Bernardini G, Santucci A: Amyloidosis, inflammation, and oxidative stress in the heart of an alkaptonuric patient. Mediators Inflamm 2014, 2014:258471

30. Millucci L, Giorgetti G, Viti C, Ghezzi L, Gambassi S, Braconi D, Marzocchi B, Paffetti A, Lupetti P, Bernardini G, Orlandini M, Santucci A: Chondroptosis in alkaptonuric cartilage. J Cell Physiol 2015, 30:1148-1157

31. Braconi D, Laschi M, Taylor AM, Bernardini G, Spreafico A, Tinti L, Gallagher JA, Santucci A: Proteomic and redox-proteomic evaluation of homogentisic acid and ascorbic acid effects on human articular chondrocytes. J Cell Biochem 2010, 111:922-932

32. Braconi D, Millucci L, Ghezzi L, Santucci A: Redox proteomics gives insights into the role of oxidative stress in alkaptonuria. Expert Rev Proteomics 2013, 10:521-535

33. Tinti L, Spreafico A, Braconi D, Millucci L, Bernardini G, Chellini F, Cavallo G, Selvi E, Galeazzi M, Marcolongo R, Gallagher JA, Santucci A: Evaluation of antioxidant drugs for the treatment of ochronotic alkaptonuria in an in vitro human cell model. J Cell Physiol 2010, 225:84-91

34. Sealock RR, Gladstone M, Steele JM: Administration of ascorbic acid to an alkaptonuric patient. Proc Soc Exp Biol Med 1940, 44:580-583

35. Braconi D, Bianchini C, Bernardini G, Laschi M, Millucci L, Spreafico A, Santucci A: Redox-proteomics of the effects of homogentisic acid in an in vitro human serum model of alkaptonuric ochronosis. J Inherit Metab Dis 2011, 34:1163-1176

36. Introne WJ, Perry MB, Troendle J, Tsilou E, Kayser MA, Suwannarat P, O'Brien KE, Bryant J, Sachdev V, Reynolds JC, Moylan E, 
Bernardini I, Gahl WA: A 3-year randomized therapeutic trial of nitisinone in alkaptonuria. Mol Genet Metab 2011, 103:307-314

37. Ranganath LR, Milan AM, Hughes AT, Dutton JJ, Fitzgerald R, Briggs MC, Bygott $\mathrm{H}$, Psarelli EE, Cox TF, Gallagher JA, Jarvis JC, van Kan C, Hall AK, Laan D, Olsson B, Szamosi J, Rudebeck M, Kullenberg T, Cronlund A, Svensson L, Junestrand C, Ayoob H, Timmis OG, Sireau N, Le Quan Sang KH, Genovese F, Braconi D, Santucci A, Nemethova M, Zatkova A, McCaffrey J, Christensen P, Ross G, Imrich R, Rovensky J: Suitability Of Nitisinone In Alkaptonuria 1 (SONIA 1): an international, multicentre, randomised, open-label, no-treatment controlled, parallel-group, dose-response study to investigate the effect of once daily nitisinone on 24-h urinary homogentisic acid excretion in patients with alkaptonuria after 4 weeks of treatment. Ann Rheum Dis 2016, 75: 362-367

38. Nemethova M, Radvanszky J, Kadasi L, Ascher DB, Pires DE, Blundell TL, Porfirio B, Mannoni A, Santucci A, Milucci L, Sestini S, Biolcati G, Sorge F, Aurizi C, Aquaron R, Alsbou M, Lourenço CM,
Ramadevi K, Ranganath LR, Gallagher JA, van Kan C, Hall AK, Olsson B, Sireau N, Ayoob H, Timmis OG, Sang KH, Genovese F, Imrich R, Rovensky J, Srinivasaraghavan R, Bharadwaj SK, Spiegel R, Zatkova A: Twelve novel HGD gene variants identified in 99 alkaptonuria patients: focus on "black bone disease" in Italy. Eur J Hum Genet 2016, 24:66-72

39. Laschi M, Bernardini G, Dreassi E, Millucci L, Geminiani M, Braconi D, Marzocchi B, Botta M, Manetti F, Santucci A: Inhibition of para-hydroxyphenylpyruvate dioxygenase by analogues of the herbicide nitisinone as a strategy to decrease homogentisic acid levels, the causative agent of alkaptonuria. ChemMedChem 2016, 11:674-678

40. Chaabo K, Kirkham B: Rheumatoid arthritis: anti-TNF. Int Immunopharmacol 2015, 27:180-184

41. Cummings SR, San Martin J, McClung MR, Siris ES, Eastell R, Reid IR, Delmas P, Zoog HB, Austin M, Wang A, Kutilek S, Adami S, Zanchetta J, Libanati C, Siddhanti S, Christiansen C: Denosumab for prevention of fractures in postmenopausal women with osteoporosis. $\mathrm{N}$ Engl J Med 2009, 361:756-765 